\title{
Molecular screening of multidrug-resistance tuberculosis by a designated public health laboratory in Taiwan
}

\author{
H.-C. Lin ${ }^{1,2}$ • C.-L. Perng ${ }^{2}$ - Y.-W. Lai ${ }^{2}$ - F.-G. Lin ${ }^{3}$ - C.-J. Chiang ${ }^{4}$ - H.-A. Lin ${ }^{5}$ • \\ R. Jou ${ }^{6,7} \cdot$ T.-S. Chiueh ${ }^{1,2,8}$
}

Received: 15 May 2017 / Accepted: 31 July 2017 /Published online: 24 August 2017

(C) The Author(s) 2017. This article is an open access publication

\begin{abstract}
This manuscript describes our experience in early identifying MDR-TB cases in high-risk populations by setting up a single-referral molecular diagnosis laboratory in Taiwan. Taiwan Centers for Disease Control designated a singlereferral laboratory to provide the GenoType MTBDRplus test for screening high-risk MDR-TB populations nationwide in 2012-2015. A total of 5,838 sputum specimens from 3,308 patients were tested within 3 days turnaround time. Compared with the conventional culture and drug susceptibility testing, the overall performance of the GenoType MTBDRplus test for detecting TB infection showed accuracy of $70.7 \%$, sensitivity of $85.9 \%$, specificity of $65.7 \%$, positive predictive value of
\end{abstract}

R. Jou

rwj@cdc.gov.tw

$\triangle$ T.-S. Chiueh

hsinchunglin@gmail.com

1 Graduate Institute of Medical Sciences, National Defense Medical Center, Taipei, Taiwan, Republic of China

2 Division of Clinical Pathology, Department of Pathology, Tri-Service General Hospital, Taipei, Taiwan, Republic of China

3 School of Public Health, National Defense Medical Center, Taipei, Taiwan, Republic of China

4 Chest Hospital, Ministry of Health and Welfare, Tainan, Taiwan, Republic of China

5 Division of Infection, Department of Medicine, Tri-Service General Hospital SongShan Branch, Taipei, Taiwan, Republic of China

6 Tuberculosis Research Center, Taiwan Centers for Disease Control, No. 161, Kun-Yang Street, Taipei 11561, Republic of China

7 Institute of Microbiology and Immunology, National Yang-Ming University, Taipei, Taiwan, Republic of China

8 Department of Laboratory Medicine, Linkou Chang Gung Memorial Hospital, No. 5 Fu-Hsing Street, Kuei-Shan, TaoYuan

City 33305, Taiwan, Republic of China
$45.5 \%$, and negative predictive value of $93.3 \%$. And the accuracy of detecting rifampin (RIF) resistance, isoniazid (INH) resistance, and MDR-TB (resistant to at least RIF and INH) were $96.5 \%, 95.2 \%$, and $97.7 \%$, respectively. MDR-TB contacts presented a higher rate of mutated codons 513-519, GenoType MTBDRplus banding pattern: $r р о B$ WT3(-), and $r р о B$ WT4(-) than the treatment failure group. The MDR-TB contact group also had a higher rate of inhA C15T mutation, banding pattern: inhA WT1(-), and inhA MUT1(+) than the recurrent group. Resistance profiles of MDR-TB isolates also varied geographically. The referral molecular diagnosis system contributed to rapid detection and initiation of appropriate therapy.

\section{Introduction}

Tuberculosis (TB) is a major health challenge in Taiwan, where it is endemic [1]. The resurgence of TB in recent years has been shown to correlate with an increase in M. tuberculosis isolates which are resistant to one or more of the first-line treatment drugs [2]. A hospital-based study which evaluated 1961 Taiwanese TB patients between 2003 and 2007 showed that $11.7 \%$ of the patients were resistant to $\mathrm{INH}, 2.8 \%$ were resistant to RIF, $2.5 \%$ were resistant to $\mathrm{EMB}$, and $11.1 \%$ were resistant to streptomycin (SM). The overall resistance to any drug was $19.1 \%$, while $2 \%$ were multidrug-resistant (MDR; defined as resistant to at least INH and RIF) [3]. Other studies from Taiwan have shown that $10.1 \%$ of the M. tuberculosis strains were resistant to INH, $6.2 \%$ were resistant to RIF, $2.1 \%$ were resistant to $\mathrm{EMB}$, and $9.8 \%$ were resistant to SM. In addition, $18.1 \%$ of these strains were resistant to any of the first-line drugs, and 4\% were MDR [4-6]. The rates of MDR-TB among new cases and previously treated cases in Taiwan were $1.1 \%$ and 6-7\% respectively [7]. 
The Taiwan Centers for Disease Control (TCDC) in accordance with WHO recommendations, implemented an MDR-TB management program (DOTS-plus program) in 2007 [4, 8]. However, the current standard care for MDR-TB has been shown to be associated with the emergence of extensively drug-resistant (XDR) TB [9, 10]. Earlier detection of MDR-TB cases and prompt initiation of appropriate treatment was therefore necessary for achieving the goal of DOTS-plus program.

Previous studies demonstrate drug resistance mutations of rpoB, inhA, and katG genes in M. tuberculosis [11-13]. Sequence analysis of the $r p o B$ gene from 68 isolates demonstrated mutations in codons 531 (63.6\%, TCG/TTG; 4.5\%, TCG/TGG), 513 (9.1\%, CAA/AAA), 533 (9.1\%, CTG/ CCG), 516 (4.5\%, GAC/GTC), and 526 (4.5\%, CAC/TGC) [14]. The most common $k a t G$ mutations found in Taiwan were Arg463Leu (51\%), Ser315Thr (29\%), Ser315Asn (9.8\%), and other loci $(22 \%)$. However, the frequency of inhA gene mutations was low (2.4\%) [15].

Recent advances in molecular techniques facilitate identifying M. tuberculosis and determining its drug susceptibility directly from clinical specimens [16-21]. The Xpert MTB/ RIF system is an automated molecular test to detect M. tuberculosis and determine RIF resistance directly from sputum [22]. The GenoType MTBDRplus test recommended by WHO has been extensively used to detect $M$. tuberculosis complex and determine its resistance to RIF and INH [13, 18, 19, 23, 24]. The GenoType MTBDRplus test uses a combination of PCR amplification and reverse blotting with specific probes blotted on nitrocellulose strips to detect mutations of the rpoB (D516V, H526Y, H526D, S531 L), katG (S315 T), and inhA (C15T, A16G, T8C, and T8A) genes. Drug resistance is defined by the loss of any wild type probe's signal or the presence of any mutant probe's signal.

Although nucleotide mutations of the $r p o B, k a t G$, and $i n h A$ genes were previously shown to be associated with RIF and INH resistance respectively, there are currently no reports describing the correct interpretation of different banding patterns for this test. Additionally, since current published data on diagnostic performance of the GenoType MTBDRplus test are often limited in sample size, the diagnostic value of the test remains unclear in the general population or in specific groups of interest. In this study, we aimed to evaluate whether the GenoType MTBDRplus test is an adequate rapid test for diagnosis of pulmonary $\mathrm{TB}$, and to determine drug resistance in TB high-risk populations.

\section{Methods}

\section{Patient enrollment}

We enrolled high-risk of MDR-TB individuals including default, treatment failure, relapse cases, inhabitants of aboriginal villages (Zhuoxi, Wanrong, and Xiulin villages in Hualien County) and suspects from the high TB burden counties (Lunbei in Yunlin County), MDR-TB contacts, and suspects staying in high MDR-TB burden countries for more than 1 month in the preceding year. A total of 5,838 sputum specimens were collected from 3,308 individual patients suspected with MDR-TB in Taiwan from March 2012 to December 2014. All specimens were subjected to acid-fast bacilli (AFB) staining, mycobacterial culture, and subsequent identification and drug susceptibility testing (DST) in authorized TB laboratories. Aliquots of the sputum sediments after NALC-NaOH ( $N$-acetyl $L$-cysteine sodium hydroxide) decontamination were sent to the referral laboratory at Tri-Service General Hospital for molecular testing using the GenoType MTBDRplus test. All test results and patient data were uploaded to the TCDC website.

\section{Detection methods}

\section{Smear microscopy}

Concentrated sputum smears were prepared by Petroff's method. Both auramine O (AO) fluorescent dye and acidfast stains were executed. Smear results were interpreted according to the guideline issued by the TCDC.

\section{Isolation of M. tuberculosis and drug susceptibility testing (DST)}

Both conventional solid agar and mycobacteria growth indicator tube (MGIT; Becton Dickinson, Sparks, MD, USA) media were used for isolation, and either conventional solid agar or MGIT was used for DST as previously described [25]. Detection of the MPB64 antigen and DST were performed as previously described [26, 27]. The sample was classified as drug resistant when the total number of colonies on the drug-containing medium were greater than $1 \%$ of the total number of colonies on the drug-free medium.

Growth of mycobacterial liquid cultures from sputum specimens was detected using an automated MGIT 960 apparatus (Becton Dickinson, Sparks, MD, USA) according to the manufacturer's instructions. Positive cultures were evaluated by standard DST with INH and RIF using the MGIT 960 IR kit (Becton Dickinson) following the manufacturer's instruction as previously described [28].

The GenoType MTBDRplus test for detecting drug resistance

Genomic bacterial DNA was extracted from $0.1 \mathrm{ml}$ of the decontaminated sputum resuspension using the GenoLyse kit (Hain Lifescience, GmbH, Nehren, Germany) according 
to the manufacturer's instructions. The PCR-amplified product DNA was used as a template in the GenoType MTBDRplus test (Hain Lifescience GmbH, Germany) which is based on reverse hybridization and an enzymatic color reaction. Drug resistance was interpreted according to the manufacturer's instructions.

\section{Entry of results}

Test results of non-MDR-TB were immediately reported on-line to the Infectious Disease Notification System of TCDC, since this national program project was conducted under commission to TCDC Ministry of Health and Welfare. Images of all GenoType MTBDRplus test strips were immediately scanned and emailed to TCDC for the second interpretation without annotation. Conventional mycobacterial culture and DST results were entered by the authorized TB laboratories to the Infectious Disease Notification System.

\section{Statistical analysis}

Data for baseline patient characteristics were described as counts with percentages, except for age which was presented as mean and standard deviation. The multiple comparisons of percentages between pair-wise groups of various locations or reasons of screening were performed using the two-proportion-Z-test with Bonferroni correction. All hypothesis tests were two-sided with a significance level of 0.05 . The results of the GenoType MTBDRplus test were summarized based on sensitivity, specificity, positive predictive value (PPV), negative predictive value (NPV), and accuracy. All statistical assessments were performed with the IBM SPSS software, version 22 (IBM Corp., Armonk, NY, USA).

\section{Results}

\section{Characteristics of the screened subjects}

More than one specimen from some patients were sent for examination in succession. MDR-TB cases were, however, only reported by individual patient instead of all specimens to avoid repetitive count on case number. Quality of specimens did result in variable results for some patients. Either discrepant negative result or repetitive positive result of the same patient was therefore omitted. No discrepant banding patterns of drug resistance were observed in specimens from the same individual. Finally, 3,308 cases were analyzed despite 5,838 specimens being tested.
Characteristics of screened individuals are summarized in Table 1 . The average age of cases was $61.5 \pm 17.3$ years old. Most of specimens were collected from males $(76.9 \%, 2,545 /$ $3,308)$. A majority of specimens were from relapse $(46.9 \%$, $1,552 / 3,308)$ and treatment failure $(35.2 \%, 1,166 / 3,308)$ cases. Of the 3,308 screened cases, $23.0 \%$ were smear negative, and $9.9 \%, 34.7 \%, 15.5 \%, 7.7 \%$, and $9.2 \%$ showed smear titers of scanty, $1+, 2+, 3+$, and $4+$, respectively. A total of 827 (25\%) cases were M. tuberculosis culture positive.

\section{M. tuberculosis isolation among the screened populations}

M. tuberculosis isolation rates were $18.9 \%(242 / 1,278)$, $28.8 \%(302 / 1,047)$, and $28.8 \%(283 / 1,046)$ in 2012,2013 , and 2014, respectively (Table 2). The relapse group had a significantly higher isolation rate than the treatment failure group (29.4\% vs $7.3 \%$ ), but a lower rate than the aboriginal inhabitants groups, the group from high MDR-TB burden countries, the default group, and MDR-TB contacts $(29.4 \%$ vs $41.7 \%, 78.9 \%, 43.5 \%$, and $57.6 \%)(p<0.05)$. The treatment failure group had the lowest isolation rate among all groups $(p<0.05)$.

\section{Comparison of GenoType MTBDRplus test results with conventional mycobacterial DST results}

Using the conventional mycobacterial isolation results as gold standard, the GenoType MTBDRplus test detected TB with $85.9 \%, 65.7 \%, 45.5 \%, 93.3 \%$, and $70.7 \%$ of sensitivity, specificity, PPV, NPV, and accuracy, respectively (Table 3).

The sensitivity, specificity, PPV, NPV, and accuracy of the GenoType MTBDRplus test were 92.1\%, 97.3\%, 85.3\%, $98.6 \%$, and $96.5 \%$, respectively for detecting RIF resistance; were $77.9 \%, 99.8 \%, 99.1 \%, 94.6 \%$, and $95.2 \%$, respectively for detecting INH resistance; and were $82.7 \%, 99.7 \%, 97.1 \%$, $97.7 \%$, and $97.7 \%$, respectively for detecting MDR (Table 4).

\section{Associations of $r p o B$ mutations with reason for screening and geographic area}

Analysis of the M. tuberculosis genotype from each group and location showed that patients in the MDR contact group had a significantly higher proportion of rpoB WT3(-) and rpoB WT4(-) cases $(33.3 \%$ and $28.6 \%$ respectively) compared to the treatment failure group (both $0 \%)$. Patients in the aboriginal inhabitants group had a significantly higher proportion of rров WT8 (-/weak) $(100 \%)$ compared to patients in the treatment failure group $(45.5 \%)$. Additionally, there was a significantly lower proportion of rpoB MUT3 (+) mutations in the south $(35.1 \%)$ compared to the north $(68.8 \%)$, east $(77.8 \%)$, and central areas $(50 \%)$. There was a 
Table 1 Characteristics of 3308 individuals suspected to have MDR-TB

\begin{tabular}{lll}
\hline & & $N=3,308$ cases \\
\hline $\begin{array}{ll}\text { Subject characteristics } \\
\text { Age (years)* }\end{array}$ & & \\
Gender & Male & $61.5 \pm 17.3$ \\
& Female & $2,545(76.9 \%)$ \\
Reason for screening & Relapse & $763(23.1 \%)$ \\
& Treatment failure & $1,552(46.9 \%)$ \\
& Aboriginal inhabitants & $1,166(35.2 \%)$ \\
& Individual from high MDR-TB burden countries & $379(11.5 \%)$ \\
& Default (loss to follow-up) & $76(2.3 \%)$ \\
AFB Smear titer & MDR-TB contacts & $69(2.1 \%)$ \\
& Negative & $66(2.0 \%)$ \\
& Scanty & $762(23.0 \%)$ \\
& $1+$ & $326(9.9 \%)$ \\
& $2+$ & $1,147(34.7 \%)$ \\
& $3+$ & $513(15.5 \%)$ \\
& $4+$ & $255(7.7 \%)$ \\
& & $305(9.2 \%)$ \\
\hline
\end{tabular}

Data are presented as counts and percentages except for *age which is presented as mean \pm standard deviation AFB, acid fast bacilli

$\dagger$ M. tuberculosis culture (+) among screened cases significantly higher proportion of $\operatorname{rpoB} \mathrm{WT} 8(+)$ in the south compared to the north (Table 5).

\section{Associations of $\operatorname{inhA} / \mathbf{k a t G}$ mutations with reason for screening and gengraphic area}

Patients in the MDR contact group had a significantly higher proportion of inhA MUT1(+) cases (47.8\%) compared to patients in the recurrent group (18.2\%). However, the MDR contact group had a significantly lower proportion of inhA WT1(+) cases (47.8\%) compared to patients in the recurrent group (83.6\%). Interestingly, there were no significant differences in the prevalence of the $\operatorname{inh} \mathrm{A} / k a t \mathrm{G}$ mutations in the different geographical areas (Table 6).

\section{Discussion}

Although clinical practice guidelines have recommended the routine use of nucleic-acid amplification testing for evaluating MDR-TB since 1996, clinicians and laboratory personnel have not implemented the recommendation widely because of the cost. Taiwan is a moderate-TB-burden country (annual TB incidence: $49.4 / 100,000$ population in 2015), with approximately 120 new MDR-TB cases reported annually. It has been reported that $1.1 \%$ of the Taiwanese annual new TB cases has MDR-TB. Recent data have shown that MDR status was significantly associated with poor long-term outcome, and MDR-TB patients had significantly shorter survival times compared to non-MDR-TB patients [29]. Screening only high-risk patients with the GenoType MTBDRplus test would significantly reduce the cost burden, since patients without

Table 2 Isolation rates of $M$. tuberculosis among 3,308 screened populations by group

\begin{tabular}{|c|c|c|c|c|c|c|c|}
\hline Year & Total & Relapse & $\begin{array}{l}\text { Treatment } \\
\text { failure }\end{array}$ & $\begin{array}{l}\text { Aboriginal } \\
\text { inhabitants }\end{array}$ & $\begin{array}{l}\text { Individual from } \\
\text { high MDR-TB } \\
\text { burden countries }\end{array}$ & $\begin{array}{l}\text { Default } \\
\text { (Loss to } \\
\text { follow-up) }\end{array}$ & $\begin{array}{l}\text { MDR-TB } \\
\text { contacts }\end{array}$ \\
\hline Total & $25 \%(827 / 3,308)$ & $29.4 \%(456 / 1,552)$ & $7.3 \%(85 / 1,166)$ & $41.7 \%(158 / 379)$ & - & $34.2 \%(13 / 38)$ & $57.6 \%(38 / 66)$ \\
\hline Year 2012 (March to Dec.) & $18.9 \%(242 / 1,278)$ & $20.8 \%(129 / 620)$ & $8.1 \%(30 / 370)$ & $25.7 \%(56 / 218)$ & & & $43.8 \%(14 / 32)$ \\
\hline Year 2013 & $28.8 \%(302 / 1,047)$ & $33.1 \%(164 / 496)$ & $9.3 \%(36 / 389)$ & $57.3 \%(55 / 96)$ & $83.9 \%(26 / 31)$ & $44.4 \%(8 / 18)$ & $76.5 \%(13 / 17)$ \\
\hline Year 2014 & $28.8 \%(283 / 983)$ & $37.4 \%(163 / 436)$ & $4.7 \%(19 / 407)$ & $72.3 \%(47 / 65)$ & $75.6 \%(34 / 45)$ & $69.2 \%(9 / 13)$ & $64.7 \%(11 / 17)$ \\
\hline
\end{tabular}

Data are represented as percent $(n / N)$ for given specific screening reason and time period. $n$, number of subjects with TB isolation $(+)$; $N$, total subjects for given specific group and time period 
Table 3 Performance of the GenoType MTBDRplus test $(n=3308)$

\begin{tabular}{lllllllll}
\hline GenoType MTBDRplus & \multicolumn{2}{l}{ M. tuberculosis isolation } & Sen. & Spe. & PPV & NPV & Accuracy \\
\cline { 2 - 6 } & & Yes & No & & & & & \\
\hline TB & Yes & 710 & 851 & $85.9 \%$ & $65.7 \%$ & $45.5 \%$ & $93.3 \%$ & $70.7 \%$ \\
& No & 117 & 1,630 & & & & & \\
\hline
\end{tabular}

Abbreviations: TB, tuberculosis; Sen., sensitivity; Spe., specificity; PPV, positive predictive value; NPV, negative predictive value; MDR, multidrug resistance significant risk factors can continue to be screened using traditional methods. We therefore established a referral molecular diagnosis laboratory for nation-wide screening of MDRTB using a cost-effective strategy. Our data were consistent with previous studies on the performance of the GenoType MTBDRplus tests [13, 18, 19, 23, 24, 29].

Although mycobacterial culture and conventional DST remain the gold standards for diagnosis of TB and determining drug susceptibility, they are so time-consuming and technically demanding that effective and timely treatment can be compromised. The GenoType MTBDRplus test enables rapid diagnosis of TB and detection of INH and or RIF resistance. Drug resistance data showed that the GenoType MTBDRplus test was highly consistent with conventional DST assays [30-33], and the results could be reported within 3 working days. Earlier clinical management of patients identified with MDR-TB could certainly reduce the risk of transmission. Additionally, recent studies demonstrated the use of the GenoType MTBDRplus test to confirm the presence of M. tuberculosis in HIV-infected patients, and in AFB-negative smears with positive TB-PCR results [34, 35]. Single drug-resistant mycobacteria are mostly not cultivatable because of inhibition by the residual anti-TB drugs in specimens, and administration of effective treatment can be compromised. Negligence of the mono-resistant mycobacteria could potentially result in selecting for MDR-TB due to insufficient efficacy of the combined regimen. Indeed, erroneous treatment strategies and previous TB therapy were recently shown to be highly correlated with acquisition of MDR-TB, and each additional treatment episode doubled the risk of MDR-TB [36].

Rapid diagnosis of MDR-TB using molecular methods was therefore introduced for blocking secondary transmission as early as possible. In this study, rapid molecular testing yielded positive results in 851 cases $(25.7 \%)$ which were negative by a conventional culture method. This might be because patients were receiving therapy, or because of low bacilli load in the specimens. Additionally, a total of 11 MDR-TB cases were positive by molecular rapid testing but negative by conventional culture. This could be due to the low concentration drug resistance missed by conventional DST. Furthermore, 117 cases which were negative by molecular rapid testing but positive by conventional culture had scanty smear results. This might be due to the detection limit of the GenoType MTBDRplus test, and is an inherent disadvantage of this generation of the GenoType MTBDRplus test, which can only be used in specimens with positive sputum smears. Therefore, drug-resistant gene sequencing is recommended to confirm wild-type results which may be missed by the GenoType MTBDRplus test.

A major advantage of the GenoType MTBDRplus test is its ability to detect mutations associated with RIF and INH resistance. A previous study which detected the presence of $r p o B$, inhA, and kat $G$ mutations using the GenoType MTBDRplus test reported the benefits of using this assay as an initial screen to reduce the delay in initiating treatment, and to guide the
Table 4 The performance of the GenoType MTBDRplus test in predicting mycobacterial drug resistances $(n=710 *)$

\begin{tabular}{|c|c|c|c|c|c|c|c|c|}
\hline \multicolumn{2}{|c|}{ GenoType MTBDRplus } & \multicolumn{2}{|c|}{$\begin{array}{l}\text { Corresponding } \\
\text { DST results† }\end{array}$} & \multirow[t]{2}{*}{ Sen. } & \multirow[t]{2}{*}{ Spe. } & \multirow[t]{2}{*}{ PPV } & \multirow[t]{2}{*}{ NPV } & \multirow[t]{2}{*}{ Accuracy } \\
\hline & & $\mathrm{R}$ & $\mathrm{S}$ & & & & & \\
\hline RIF resistance & $\begin{array}{l}\mathrm{R} \\
\mathrm{S}\end{array}$ & $\begin{array}{r}93 \\
8\end{array}$ & $\begin{array}{r}16 \\
567\end{array}$ & $92.1 \%$ & $97.3 \%$ & $85.3 \%$ & $98.6 \%$ & $96.5 \%$ \\
\hline INH resistance & $\begin{array}{l}\mathrm{R} \\
\mathrm{S}\end{array}$ & $\begin{array}{r}109 \\
31\end{array}$ & $\begin{array}{r}1 \\
543\end{array}$ & $77.9 \%$ & $99.8 \%$ & $99.1 \%$ & $94.6 \%$ & $95.2 \%$ \\
\hline MDR & $\begin{array}{l}\mathrm{R} \\
\mathrm{S}\end{array}$ & $\begin{array}{l}67 \\
14\end{array}$ & $\begin{array}{r}2 \\
601\end{array}$ & $82.7 \%$ & $99.7 \%$ & $97.1 \%$ & $97.7 \%$ & $97.7 \%$ \\
\hline
\end{tabular}

*710 patients with Genotype TB (+) and culture M. tuberculosis isolation (+)

†Only 684 patients with completed DST results

Abbreviations: R, resistance; S, susceptible; Sen., sensitivity; Spe., specificity; PPV, positive predicted value; NPV, negative predicted value; INH, isoniazid, RIF, rifampicin; MDR, multidrug resistance to at least INH and RIF 


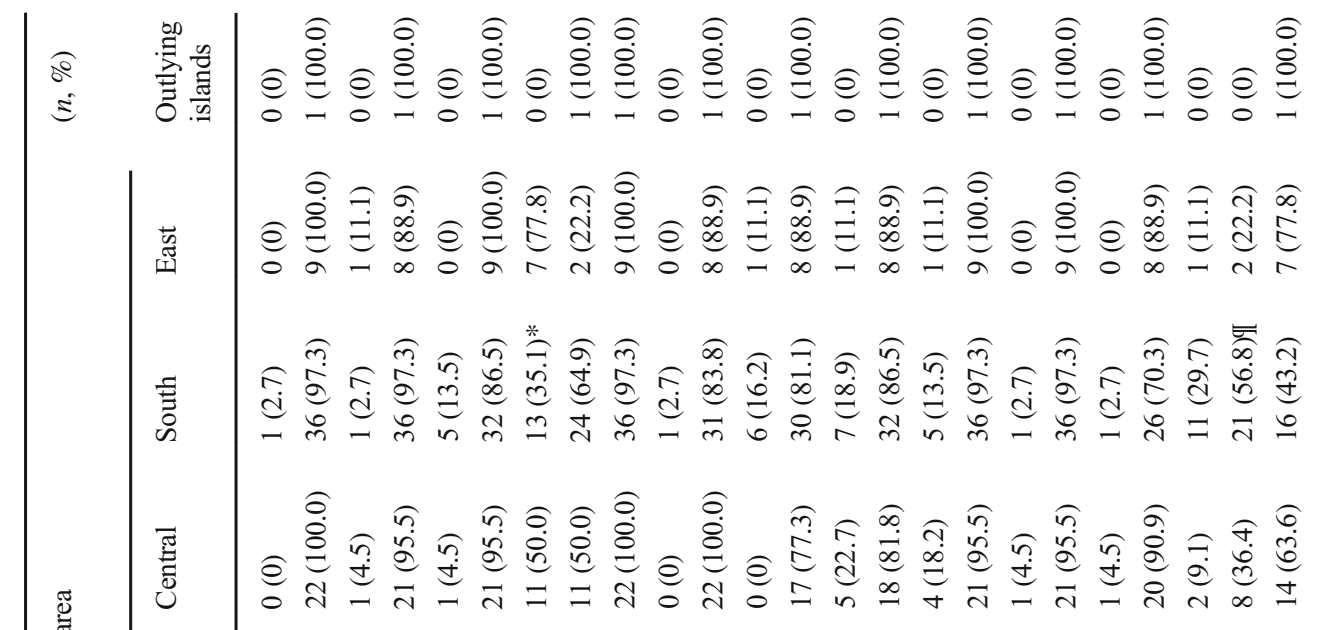

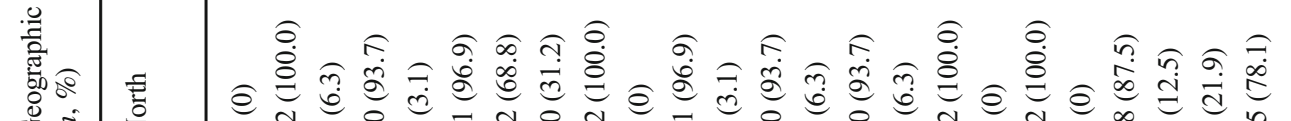

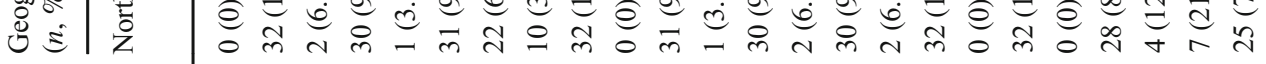

흐

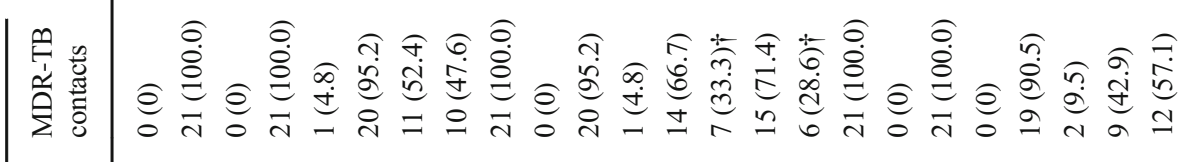
a

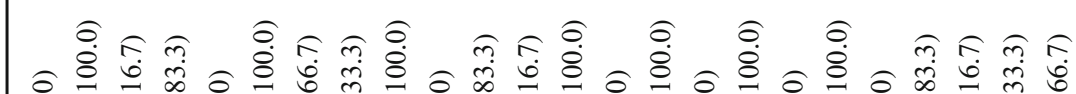

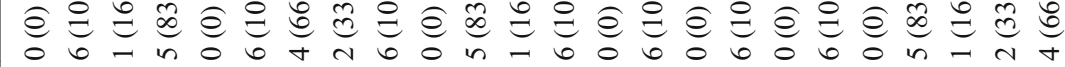

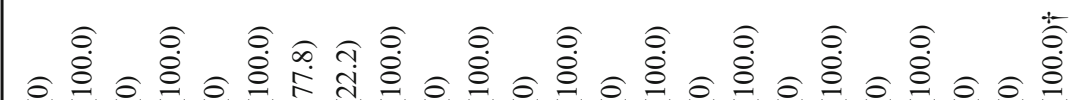

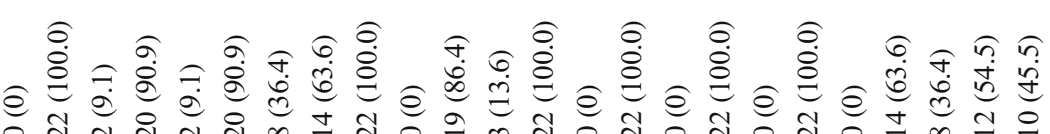




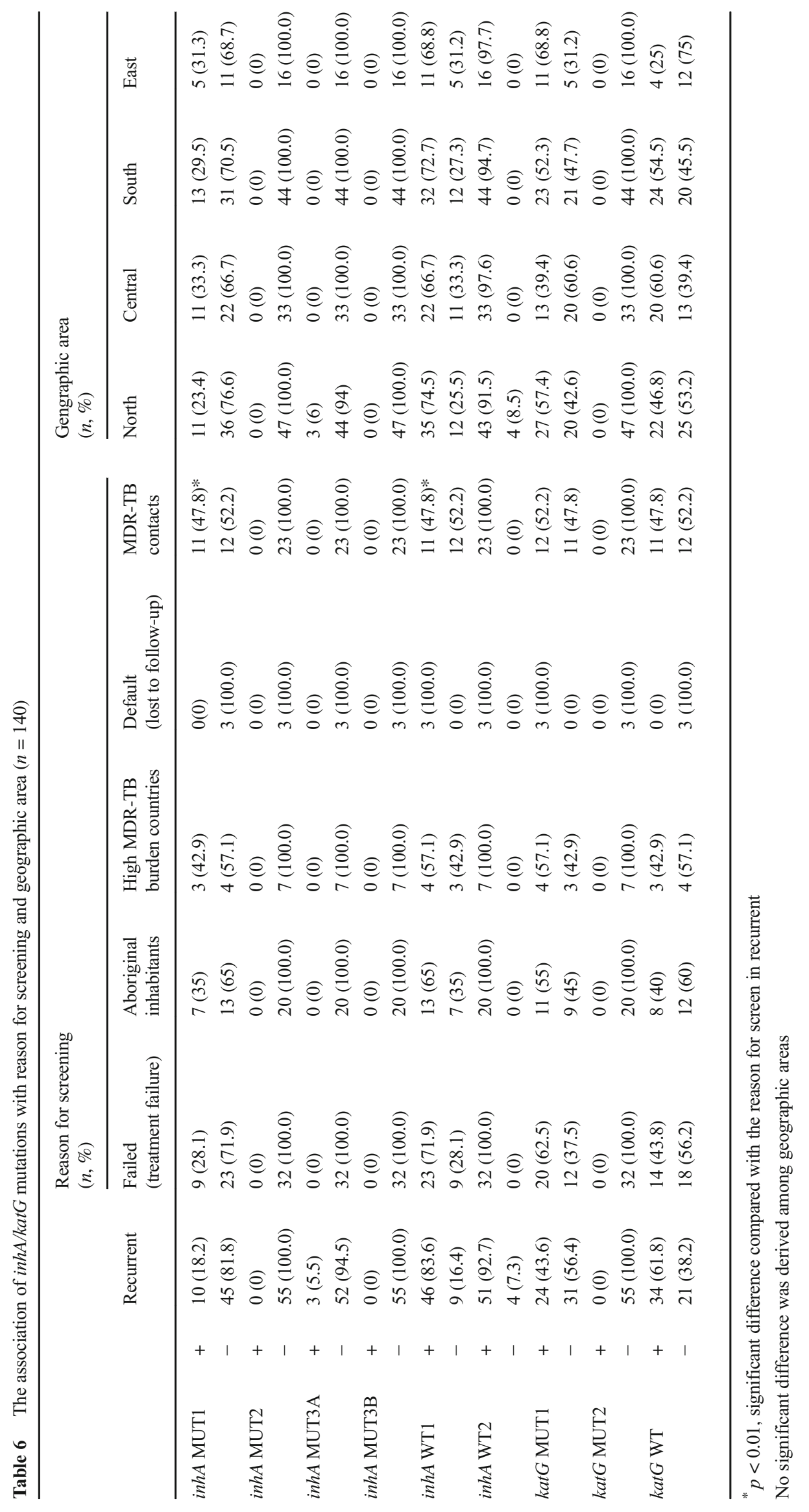


recommendation for first- and second-line DST [37]. Early initiation of an appropriate treatment regimen is especially important, since this may be a critical factor to reduce the number of patients lost to follow-up [38]. However, to the best of our knowledge, ours is the first study to analyze the association between rpoB, $i n h A$, and $k a t G$ mutations and the reason for screening, as well as geographical area. Our data showed that patients in the MDR contact group had a higher proportion of $r p o B$ WT3(-) and $r p o B$ WT4(-) (denoting mutations in codons 513-519), and patients in the aboriginal inhabitants group had a higher proportion of $r p o B$ WT8 (-) (denoting mutations in codons 530-533) compared to the treatment failure group. The MDR contact group also had a lower prevalence of inhA WT1(+) and a higher prevalence of inhA MUT1(+) (denoting a C15T mutation) compared to the recurrent group. Analysis of mutations associated with location showed that there was a significantly lower proportion of $r p o B$ MUT3(+) and a significantly higher proportion of rpoB WT8(+) mutations in the south compared to the north.

\section{Conclusions}

We demonstrated the diagnostic value of the GenoType MTBDRplus test for detection of MDR-TB. In addition to its high accuracy, the major advantage of the GenoType MTBDRplus test is the ability to reduce possible secondary transmission of MDR-TB by virtue of earlier diagnosis with satisfactory sensitivity. This system is still being implemented, and provides MDR-TB identification service nation-wide. We expect that it will help to reduce the annual incidence of MDR$\mathrm{TB}$ in Taiwan.

Authors' contributions Hsin-Chung Lin: definition of intellectual content; literature research; clinical studies; experimental studies; data acquisition; data analysis; statistical analysis; manuscript preparation.

C-L P: experimental studies;

Y-W L: experimental studies; data acquisition.

F-G L: statistical analysis.

C-J C: experimental studies.

H-A L: literature research; clinical studies; data analysis; statistical analysis.

R J: study concepts; study design; manuscript editing; manuscript review.

T-S C: guarantor of integrity of the entire study; study concepts; study design; definition of intellectual content; manuscript editing; manuscript review.

All authors have read and approved the final version for publication.

Funding This study was supported by a grant from Taiwan Centers for Disease Control and Tri-Service General Hospital, Taiwan, Republic of China (grant nos. TSGH-C103-193, TSGH-C105-188, TSGHSBC104-06, and TSGHSB-C105-01).

\section{Compliance with ethical standards}

Conflict of interest The authors declare that they have no conflict of interest.
Ethical approval This study was approved by the Institutional Review Board II of the Tri-Service General Hospital.

Informed consent All subjects gave their written informed consent prior to participation in the study.

Open Access This article is distributed under the terms of the Creative Commons Attribution 4.0 International License (http:// creativecommons.org/licenses/by/4.0/), which permits unrestricted use, distribution, and reproduction in any medium, provided you give appropriate credit to the original author(s) and the source, provide a link to the Creative Commons license, and indicate if changes were made.

\section{References}

1. Chan PC, Chen CH, Chang FY (2014) Extended review of the National Tuberculosis Program and the development of strategy and targets post 2015 in Taiwan. J Formos Med Assoc 113:775-777

2. Bloch AB, Cauthen GM, Onorato IM, Dansbury KG, Kelly GD, Driver CR, Snider DE Jr (1994) Nationwide survey of drugresistant tuberculosis in the United States. JAMA 271:665-671

3. Yu CC, Chang CY, Liu CE, Shih LF, Hsiao JH, Chen CH (2010) Drug resistance pattern of mycobacterium tuberculosis complex at a medical center in central Taiwan, 2003-2007. J Microbiol Immunol Infect 43:285-290

4. Chang CW, Wu MH, Chuang PC, Jou R (2011) Characteristics of multidrug-resistant mycobacterium tuberculosis in Taiwan: a population-based study. Infect Genet Evol 11:633-639

5. Su WJ (2008) Extensively drug-resistant tuberculosis (XDR-TB) raises challenges in TB control in Taiwan. J Formos Med Assoc 107:827-829

6. Yu MC, Wu MH, Jou R (2008) Extensively drug-resistant tuberculosis, Taiwan. Emerg Infect Dis 14:849-850

7. Centers for Disease Control ROCT (2011) Taiwan Tuberculosis Control Report, 2011. Ministry of Health and Welfare, Taiwan

8. Yu MC, Chen HY, Chien SH, Jou R (2015) An integrated management program for MDR-TB results in favourable outcomes in northern Taiwan. Eur Respir J 45:272-275

9. Brossier F, Veziris N, Aubry A, Jarlier V, Sougakoff W (2010) Detection by GenoType MTBDRsl test of complex mechanisms of resistance to second-line drugs and ethambutol in multidrugresistant mycobacterium tuberculosis complex isolates. J Clin Microbiol 48:1683-1689

10. Kliiman K, Altraja A (2009) Predictors of poor treatment outcome in multi- and extensively drug-resistant pulmonary TB. Eur Respir J 33:1085-1094

11. Musser JM (1995) Antimicrobial agent resistance in mycobacteria: molecular genetic insights. Clin Microbiol Rev 8:496-514

12. Musser JM, Kapur V, Williams DL, Kreiswirth BN, van Soolingen D, van Embden JD (1996) Characterization of the catalaseperoxidase gene $(\mathrm{katG})$ and inhA locus in isoniazid-resistant and susceptible strains of mycobacterium tuberculosis by automated DNA sequencing: restricted array of mutations associated with drug resistance. J Infect Dis 173:196-202

13. Huang WL, Chen HY, Kuo YM, Jou R (2009) Performance assessment of the GenoType MTBDRplus test and DNA sequencing in detection of multidrug-resistant mycobacterium tuberculosis. J Clin Microbiol 47:2520-2524

14. Lin YH, Tai CH, Li CR, Lin CF, Shi ZY (2013) Resistance profiles and $\mathrm{rpoB}$ gene mutations of mycobacterium tuberculosis isolates in Taiwan. J Microbiol Immunol Infect 46:266-270 
15. Tseng ST, Tai CH, Li CR, Lin CF, Shi ZY (2015) The mutations of katG and inhA genes of isoniazid-resistant mycobacterium tuberculosis isolates in Taiwan. J Microbiol Immunol Infect 48:249-255

16. Stop TB Partnership WHO (2006) Global Plan to Stop TB, 20062015. WHO. Available from: WHO/HTB/STB/2006

17. Cole ST, Brosch R, Parkhill J, Garnier T, Churcher C, Harris D, Gordon SV, Eiglmeier K, Gas S, Barry CE 3rd, Tekaia F, Badcock K, Basham D, Brown D, Chillingworth T, Connor R, Davies R, Devlin K, Feltwell T, Gentles S, Hamlin N, Holroyd S, Hornsby T, Jagels K, Krogh A, McLean J, Moule S, Murphy L, Oliver K, Osborne J, Quail MA, Rajandream MA, Rogers J, Rutter S, Seeger K, Skelton J, Squares R, Squares S, Sulston JE, Taylor K, Whitehead S, Barrell BG (1998) Deciphering the biology of mycobacterium tuberculosis from the complete genome sequence. Nature 393:537-544

18. Akpaka PE, Baboolal S, Clarke D, Francis L, Rastogi N (2008) Evaluation of methods for rapid detection of resistance to isoniazid and rifampin in mycobacterium tuberculosis isolates collected in the Caribbean. J Clin Microbiol 46:3426-3428

19. Barnard M, Albert H, Coetzee G, O'Brien R, Bosman ME (2008) Rapid molecular screening for multidrug-resistant tuberculosis in a high-volume public health laboratory in South Africa. Am J Respir Crit Care Med 177:787-792

20. Ong DC, Yam WC, Siu GK, Lee AS (2010) Rapid detection of rifampicin- and isoniazid-resistant mycobacterium tuberculosis by high-resolution melting analysis. J Clin Microbiol 48:1047-1054

21. Pang Y, Zhou Y, Wang S, Lu J, Lu B, He G, Wang L, Zhao Y (2011) A novel method based on high resolution melting (HRM) analysis for MIRU-VNTR genotyping of mycobacterium tuberculosis. J Microbiol Methods 86:291-297

22. Blakemore R, Story E, Helb D, Kop J, Banada P, Owens MR, Chakravorty S, Jones M, Alland D (2010) Evaluation of the analytical performance of the Xpert MTB/RIF assay. J Clin Microbiol 48:2495-2501

23. Causse M, Ruiz P, Gutierrez JB, Zerolo J, Casal M (2008) Evaluation of new GenoType MTBDRplus for detection of resistance in cultures and direct specimens of mycobacterium tuberculosis. Int J Tuberc Lung Dis 12:1456-1460

24. Hillemann D, Rüsch-Gerdes S, Richter E (2007) Evaluation of the GenoType MTBDRplus assay for rifampin and isoniazid susceptibility testing of mycobacterium tuberculosis strains and clinical specimens. J Clin Microbiol 45:2635-2640

25. WHO (1998) Laboratory services in tuberculosis contol. Culture Part III. WHO, Geneva. Available from: WHO/TB/98.258

26. Aung WW, Ei PW, Nyunt WW, Swe TL, Lwin T, Htwe MM, Kim KJ, Lee JS, Kim CK, Cho SN, Song SD, Chang CL (2015) Phenotypic and genotypic analysis of anti-tuberculosis drug resistance in mycobacterium tuberculosis isolates in Myanmar. Ann Lab Med 35:494-499

27. WHO (2003). Guidelines for surveillance of drug resistance in tuberculosis. WHO/CDS/CSR/RMD/2003. WHO, GENEVA.
Available from: http://whqlibdoc.who.int/publications/2003/ 9241546336.pdf

28. Centers for disease control (2013) Taiwan Guidelines for TB Diagnosis and Treatment. 5th Ed. Ministry of Health and Welfare, Taiwan

29. Sun Y, Harley D, Vally H, Sleigh A (2015) Comparison of characteristics and mortality in multidrug resistant (MDR) and non-MDR tuberculosis patients in China. BMC Public Health 15:1027-1034

30. Lacoma A, Garcia-Sierra N, Prat C, Ruiz-Manzano J, Haba L, Rosés S, Maldonado J, Domínguez J (2008) GenoType MTBDRplus assay for molecular detection of rifampin and isoniazid resistance in mycobacterium tuberculosis strains and clinical samples. J Clin Microbiol 46:3660-3667

31. Lyu J, Kim MN, Song JW, Choi CM, Oh YM, Lee SD, Kim WS, Kim DS, Shim TS (2013) GenoType(R) MTBDRplus assay detection of drug-resistant tuberculosis in routine practice in Korea. Int $\mathrm{J}$ Tuberc Lung Dis 17:120-124

32. Miotto P, Piana F, Penati V, Canducci F, Migliori GB, Cirillo DM (2006) Use of genotype MTBDR assay for molecular detection of rifampin and isoniazid resistance in mycobacterium tuberculosis clinical strains isolated in Italy. J Clin Microbiol 44:2485-2491

33. Singhal R, Myneedu VP, Arora J, Singh N, Bhalla M, Verma A (2015) Sarin R (2015) early detection of multi-drug resistance and common mutations in mycobacterium tuberculosis isolates from Delhi using GenoType MTBDRplus assay. Indian J Med Microbiol 33(Suppl):46-52

34. Luetkemeyer AF, Kendall MA, Wu X, Lourenço MC, Jentsch U, Swindells S, Qasba SS, Sanchez J, Havlir DV, Grinsztejn B, Sanne IM, Firnhaber C, Adult AIDS Clinical Trials Group A5255 Study Team (2014) Evaluation of two line probe assays for rapid detection of mycobacterium tuberculosis, tuberculosis (TB) drug resistance, and non-TB Mycobacteria in HIV-infected individuals with suspected TB. J Clin Microbiol 52:1052-1059

35. Lee YS, Kang HR, Lee SH, Kim Y, Kim MY, Shin JH, Moon JY, Lee HK, Park SY, Mo EK, Park YB, Moon SY, Oh M, Ko Y (2016) Diagnostic usefulness of the GenoType MTBDRplus assay for detecting drug-resistant tuberculosis using AFB smear-negative specimens with positive TB-PCR result. Infect Dis (Lond) 48:350-355

36. Ignatyeva O, Balabanova Y, Nikolayevskyy V, Koshkarova E, Radiulyte B, Davidaviciene E, Riekstina V, Jaama K, Danilovits M, Popa CM, Drobniewski FA (2015) Resistance profile and risk factors of drug resistant tuberculosis in the Baltic countries. Tuberculosis 95:581-588

37. Tolani MP, D'Souza DTB, Mistry NF (2012) Drug resistance mutations and heteroresistance detected using the genotype MTBDRplus assay and their implications for treatment outcomes in patients from Mumbai, India. BMC Infect Dis 12:9-16

38. Deshmukh RD, Dhande DJ, Sachdeva KS, Sreenivas A, Kumar AMV, Satyanarayana S, Parmar M, Moonan PK, Lo TQ (2015) Patient and provider reported reasons for lost to follow up in MDRTB treatment: a qualitative study from a drug resistant TB center in India. PLoS One 10:e135802 